\title{
Polygonal $\mathbf{N}$-vortex arrays: A Stuart model
}

\author{
Darren Crowdya) \\ Department of Mathematics 2-392, Massachusetts Institute of Technology, \\ 77 Massachusetts Avenue, Cambridge, Massachusetts 02139-4307 \\ and Department of Mathematics, Imperial College, 180 Queen's Gate, London SW7 2AZ, United Kingdom
}

(Received 30 April 2003; accepted 8 September 2003; published 29 October 2003)

\begin{abstract}
A class of exact planar solutions of the Euler equations representing stationary $N$-polygonal arrays of vortices are found. The solutions are parametrized by two parameters $N$ and $\Omega_{\max }$. N denotes the number of vorticity extrema surrounding the origin; $\Omega_{\max }$ denotes the extremal value of this vorticity. Except for a point vortex at the origin, the solutions have everywhere-smooth vorticity distributions and are generalizations of the classic exact solution of Stuart [J. Fluid Mech. 29, 417 (1967)] for an infinite row of smooth vortices. In the limit $\left|\Omega_{\max }\right| \rightarrow \infty$, the solutions reduce to the pure point vortex problem considered by Morikawa and Swenson [Phys. Fluids 14, 1058 (1971)]. The new solutions can be understood as "smoothed-out" counterparts to this point vortex problem. (C) 2003 American Institute of Physics. [DOI: 10.1063/1.1623766]
\end{abstract}

\section{INTRODUCTION}

The study of equilibrium arrays of vortices, sometimes called vortex "crystals" or "lattices," is a hydrodynamical problem of perennial interest. A recent monograph by Newton ${ }^{1}$ provides a modern and comprehensive survey of the $N$-vortex problem. ${ }^{2}$ Classical investigations include the work of Thomson ${ }^{3}$ and Havelock. ${ }^{4}$ A resurgence of interest in $\mathrm{N}$-vortex equilibria occurred when it was discovered that superfluid helium organizes itself into line-vortex structures. ${ }^{5}$ This even inspired the construction of a catalog of vortex patterns. ${ }^{6}$ More recently, interest in the $N$-vortex problem has been incited by the discovery of a new class of $N$-vortex structures commonly dubbed multipolar vortices. ${ }^{10,11}$ These are rotating coherent vortex structures characterized by a central core of one signature surrounded by a (usually symmetric) distribution of satellite vortices of opposite signature. They are found to form as a result of the nonlinear destabilization of shielded monopolar structures and the higherorder structures themselves have near-zero total circulation. Crowdy ${ }^{12}$ has identified a class of exact solutions of the planar Euler equations sharing many of the qualitative properties of these multipolar vortices. Beyond hydrodynamics, the fact that vortex lattices also emerge as self-organized structures in non-neutral plasmas has added further momentum to the study of such problems. ${ }^{7,8}$

By far, the problem of $N$-polygonal arrays of line vortices has received the most attention. Since the early work of Thomson, ${ }^{3}$ numerous investigations have been carried out. For example, in any effort to model the polar vortex, Morikawa and Swenson ${ }^{9}$ studied a generalization of Thomson's configuration in which an additional line vortex is placed at the center of an $\mathrm{N}$-polygonal array. Work in the area of planar line vortex equilibria continues (see, for example, Aref and Vainchtein ${ }^{13}$ ).

Beyond line-vortex models, much less is known and the

${ }^{a)}$ Electronic mail: d.crowdy@imperial.ac.uk literature is more recent. Dritschel ${ }^{14}$ employed a vortex-patch model to generalize the classic line-vortex problem considered by Thomson. Using a numerical method, he computed the shapes of co-rotating finite-area patches of uniform vorticity placed in an $N$-polygonal array. More recently, Crowdy ${ }^{15}$ has generalized the problem considered by Morikawa and Swenson ${ }^{9}$ and identified a class of exact solutions to the problem where a central vortex patch of distributed uniform vorticity is surrounded by $N$ co-rotating line vortices.

Apart from the Lamb dipole ${ }^{20}$ and some variants thereof (see, for example, Meleshko and van Heijst ${ }^{16}$ ), there currently appear to be no known exact solutions for a general planar $N$-polygonal array of vortices in equilibrium and having a smooth distribution of vorticity. A famous exact solution of the steady Euler equation which does possess a smooth vorticity distribution is that of Stuart. ${ }^{17}$ However, this solution describes an infinite periodic array of co-rotating vortices and is more relevant to modeling a shear layer profile than a finite vortex "crystal." Mallier and Maslowe ${ }^{18}$ have generalized Stuart's solution to an infinite row of vortices of alternating sign.

In this paper, new exact solutions representing a general $\mathrm{N}$-polygonal array of vortices are constructed using a Stuart model which follows in the spirit of the Stuart solution ${ }^{17}$ in assuming an exponential relation between the vorticity and streamfunction. The solutions are globally smooth except for a single line vortex singularity at the center of the $N$-polygonal array.

\section{MATHEMATICAL FORMULATION}

In 1967, Stuart ${ }^{17}$ introduced an exact solution of the steady planar Euler equations which have since become well-known to the fluid dynamics community as the "Stuart vortices." Stuart studied the solution as a model of the free shear layer. In this solution, the vorticity $\omega$ is exponentially related to the streamfunction $\psi$ so that 


$$
\omega=-\nabla^{2} \psi=-e^{-2 \psi} .
$$

It is well known ${ }^{1}$ that a solution of the steady incompressible Euler equations is obtained if the vorticity $\omega$ of an ideal fluid is purely a function of the streamfunction $\psi$, i.e., if

$$
\omega=h(\psi) \text {, }
$$

where $h(\psi)$ is some differentiable function of $\psi$. Mathematically, Stuart's choice of relation (1) between the vorticity and the streamfunction results in the quasi-linear elliptic partial differential equation for $\psi$ known as the Liouville equation. Stuart ${ }^{17}$ devotes a portion of his paper to a discussion of solutions of this equation.

Stuart's particular solution to (1) consists of an infinite periodic array of vortices described by the streamfunction

$$
\psi=\log \left(C \cosh y+\sqrt{C^{2}-1} \cos x\right),
$$

where $C$ is a real parameter satisfying $1 \leqslant C<\infty$. When $C$ $=1$, the solution represents a homogeneous shear layer profile in which all streamlines are parallel to the $x$-axis and the horizontal velocity varies like a hyperbolic tangent function with vertical distance $y$. In the opposite limit, $C \rightarrow \infty$, the solution reduces to an infinite row of identical point vortices separated by distance $2 \pi$, each of circulation $-4 \pi$. For all intermediate values $1<C<\infty$, the solution has the structure of an infinite row of (Kelvin) cat's-eyes ${ }^{20}$ with a smooth vorticity distribution. The parameter $C$ governs the steepness of the vorticity profile.

Adopting the Stuart model, ${ }^{17}$ we will seek solutions of

$$
\frac{\partial^{2} \psi}{\partial x^{2}}+\frac{\partial^{2} \psi}{\partial y^{2}}=\tilde{c} e^{d \psi},
$$

representing a polygonal $N$-polar array of vortices with a smooth vorticity distribution surrounding a single vorticity extremum at the center of the array. $\widetilde{c}$ and $d$ are real constants to be chosen later. It is convenient to complexify this equation (i.e., write it in characteristic coordinates) by introducing $z=x+i y$ and its complex conjugate $\bar{z}$. Then (4) can be written as

$$
\psi_{z \bar{z}}=c e^{d \psi},
$$

where $c=\tilde{c} / 4$ and subscripts denote partial derivatives.

To find vortical solutions within a Stuart model, we exploit the fact that the most general solution to (5) is known. Several forms of solution of (5) are listed in Stuart, ${ }^{17}$ one of which is

$$
\psi(x, y)=\frac{1}{d} \log \left[\frac{2 f^{\prime}(z) \bar{f}^{\prime}(\bar{z})}{-c d(1+f(z) \bar{f}(\bar{z}))^{2}}\right],
$$

where $c d<0$ and where $f(z)$ is an analytic function. Equation (6) is the form of solution to be used here. Stuart ${ }^{17}$ also records that his solution (3) — corresponding to the choices $d=-2$ and $\tilde{c}=1$ (implying $c=1 / 4$ ) — can be retrieved from (6) by the particular choice

$$
f(z)=A \tan (z / 2),
$$

where the constant $A$ is related to $C$. Appendix A gives more details. Readers interested in a derivation of (6) are referred to Crowdy ${ }^{19}$ where the most general solution of the elliptic Liouville equation is derived using elementary methods.

For purposes of constructing more general vortical solutions, we first make two important remarks on the nature of the analytic function $f(z)$.

(a) If $D$ denotes the domain in which a nonsingular solution of the Liouville equation is to be found, the function $f(z)$ need not necessarily be analytic everywhere in $D$; rather, it can admit a distribution of isolated simple pole singularities and still produce a solution of the elliptic Liouville equation (5) that is nonsingular in $D$. To see this, note that any simple pole of $f(z)$ at a point $\hat{z} \in \mathbb{C}$ would produce a double pole of $f^{\prime}(z)$ at the same point. However, the denominator in (6) contains the quantity $(1+f(z) \bar{f}(\bar{z}))^{2}$ which will also, in general, possess a second-order pole at $\hat{z}$. The second-order poles of both numerator and denominator thus conspire, in general, to produce a nonzero and nonsingular argument of the logarithm leading to a nonsingular solution for $\psi$.

(b) The derivative $f^{\prime}(z)$ must not vanish anywhere in $D$ if $\psi$ is to be a nonsingular solution there. This result is clear by inspection of the formula (6).

The author has not found these facts stated explicitly in the literature but they prove crucial in the analysis to follow. It therefore seems appropriate to emphasize them here. In accordance with the above two remarks, note that the choice of $f(z)$ given in (7) and yielding Stuart's solution has a countable infinity of simple poles in $\mathrm{C}$ (which is the domain $D$ relevant in this case). Its derivative, $(A / 2) \sec ^{2}(z / 2)$, vanishes nowhere in C. Stuart's solution is therefore a globally valid solution of the Liouville equation.

From (6) it is seen that the choice of $d$ simply rescales the vorticity everywhere in the plane. Following Stuart, ${ }^{17}$ we pick $d=-2$. The choice of $c$ is inconsequential; altering it simply changes $\psi$ by a constant which does not alter the velocity or vorticity fields. However, from (6) it is clear that $c>0$. We set $c=1$.

\section{A. $N$-vortex solutions}

To model $N$-polar vortices, we choose the function $f(z)$ be the $N$ th order polynomial,

$$
f(z)=\widetilde{a} z^{N}+\widetilde{b}
$$

where $\widetilde{a}, \widetilde{b} \in \mathbb{C}$ are complex constants. $N \geqslant 1$ is an integer. The domain $D$ of interest here is the entire complex plane $\mathbb{C}$. $f(z)$ is analytic everywhere in $D$ and, if $N>1$, its derivative has a zero of order $(N-1)$ at $z=0 . f(z)$ also has an $N$ th order pole at infinity but this singularity is not included in $D$. In accordance with the remarks made in the previous section, the corresponding solution for $\psi$ will therefore break down at $z=0$ (if $N>1$ ) but will be valid everywhere else. The singularity at $z=0$ will be examined in detail.

The substitution of (8) into (6) reveals that the solution only depends on $|\widetilde{a}|,|\widetilde{b}|$ and $\arg [\widetilde{a}-\tilde{b}]$. For this reason, with- 
out loss of generality, we choose $\widetilde{a}$ to be real, i.e., $\widetilde{a}=a$ $\in \mathbb{R}$. For the time being, $\tilde{b}$ will be taken to be generally complex. The streamfunction, valid for $z \neq 0$, is then given explicitly by

$$
\psi=-\frac{1}{2} \log \left(\frac{N^{2} a^{2} z^{N-1} \bar{z}^{N-1}}{\left(1+\left(a z^{N}+\tilde{b}\right)\left(a \bar{z}^{N}+\overline{\tilde{b}}\right)\right)^{2}}\right) .
$$

(9) fails to be a valid solution of (6) at $z=0$. However, it is important to note that

$$
\psi \sim-\frac{N-1}{2} \log z \bar{z}+\text { regular, } \quad \text { as } z \rightarrow 0 .
$$

That is, the singular part is precisely the streamfunction for a point vortex of circulation $\Gamma=2 \pi(N-1)$ at $z=0$. For a consistent solution of the Euler equation, the Helmholtz laws of vortex motion ${ }^{2}$ dictate that the non-self-induced component of the velocity field at $z=0$ must be zero. However, in this instance it is clear that this condition is automatically satisfied owing to the $N$-fold rotational symmetry of the streamfunction about the origin. It can be verified analytically.

We now examine the solutions in detail. Consider first the case $N=1$. The streamlines are given by

$$
1+a^{2}|z|^{2}+a \tilde{\bar{b}} z+a \tilde{b} \bar{z}+|\tilde{b}|^{2}=\alpha
$$

where $\alpha$ is a positive constant labeling streamline. These are clearly circles. This solution is uninteresting even though it is nonsingular everywhere (i.e., it does not have a point vortex at $z=0)$.

The solutions for $N>1$ are more interesting. Let $\tilde{b}$ $=b e^{i \phi}$ where $b \geqslant 0$ and $\phi$ are real. Since $f(z)$ has been chosen to be purely a function of $z^{N}$, the associated vorticity distribution has an $N$-fold rotational symmetry about the origin. It is found (either analytically or by plotting the streamlines) that $N$ vorticity extrema occur along the $N$ rays,

$$
\arg \left[z^{N}\right]=\pi+\phi .
$$

Owing to the rotational symmetry, it is clear that $\phi$ can be taken equal to zero without loss of generality in the physical solutions. This means that $\widetilde{b}=b \in \mathbb{R}$. A solution corresponding to a $\widetilde{b}$ with non-zero $\phi$ would simply correspond to a rotation of the solution (with $\phi=0$ ) through angle $\phi$ about the origin.

To examine the vorticity profile along one of the rays (12), let $z=s e^{i \pi / N}$ where $s \in \mathbb{R}$ and let $\Omega(s, a, b, N)$ be the vorticity along the ray $\arg [z]=\pi / N$. Note that the parameter $s$ is non-negative. Then,

$$
\Omega(s, a, b, N)=-\frac{4 N^{2} a^{2} s^{2 N-2}}{\left(1+b^{2}-2 a b s^{N}+a^{2} s^{2 N}\right)^{2}} .
$$

To find the distance $s_{\max }$ of the vorticity extremum from the origin, we seek a solution of

$$
\left.\frac{\partial \Omega(s, a, b, N)}{\partial s}\right|_{s=s_{\max }}=0 .
$$

Some algebra reveals that $s_{\max }$ satisfies

$$
(N+1) a^{2} s_{\max }^{2 N}-2 a b s_{\max }^{N}-(N-1)\left(1+b^{2}\right)=0 .
$$

This yields

$$
\begin{aligned}
s_{\max }(a, b, N)= & {\left[\frac{b}{a(N+1)}+\frac{b}{a(N+1)}\right.} \\
& \left.\times\left(1+\left(N^{2}-1\right) \frac{1+b^{2}}{b^{2}}\right)^{1 / 2}\right]^{1 / N} .
\end{aligned}
$$

(16) gives the distance $s_{\max }$ of the vorticity extrema as a function of the three parameters $a, b$ and $N$. It can be verified that the second partial derivative of $\Omega(s, a, b, N)$ with respect to $s$ is nonzero at $s=s_{\max }$ so that a genuine maximum or minimum exists there.

Given that there is no natural length scale associated with free vortical motion in the plane, it is natural to study the solutions by specifying that the distance of the vorticity extrema from the origin is unity, i.e., we set $s_{\max }=1$. This implies the following quadratic for $b$ :

$$
b^{2}+\frac{2 a}{N-1} b+\left(1-\frac{(N+1)}{(N-1)} a^{2}\right)=0
$$

which yields the explicit solution

$$
b(a, N)=-\frac{a}{N-1}+\sqrt{\frac{N^{2} a^{2}}{(N-1)^{2}}-1 .}
$$

The root corresponding to the negative square root branch has been discarded because it does not yield a non-negative $b$. (18) yields a real and non-negative solution for $b$ when $a \geqslant a_{\text {crit }}(N)$ where

$$
a_{\text {crit }}(N)=\sqrt{\frac{N-1}{N+1}} .
$$

Solution (18) ceases to be real when the discriminant of (17) vanishes. This occurs when $a=a_{*}(N)$ where

$$
a_{*}(N)=\frac{N-1}{N} .
$$

Manipulating (19) and (20) it can be shown that

$$
a_{\text {crit }}(N)=\frac{N}{\sqrt{N^{2}-1}} a_{*}(N)
$$

so that $a_{\text {crit }}(N)>a_{*}(N)$ for all $N>1$. This means that (18) gives a real non-negative solution for $b$ for all $a \geqslant a_{\text {crit }}(N)$.

Since $b$ is now a function of $a$ and $N$, in our notation we henceforth drop the explicit appearance of $b$ so that, for example, $\Omega(s, a, b, N)$ is now denoted $\Omega(s, a, N)$. It is convenient to define $\Omega_{\max }(a, N)$ to be the extremal value of the vorticity $\Omega(s, a, N)$ in (13) at $s_{\max }=1$, i.e.,

$$
\Omega_{\text {max }}(a, N) \equiv \Omega(1, a, N) .
$$

There should be no confusion in using the same notation $\Omega_{\max }$ to denote the extremal value of $\Omega(s, a, N)$ whether it be a maximum or a minimum. Explicitly, using (13),

$$
\Omega_{\max }(a, N)=-\frac{4 N^{2} a^{2}}{\left(1+b(a, N)^{2}-2 a b(a, N)+a^{2}\right)^{2}} .
$$




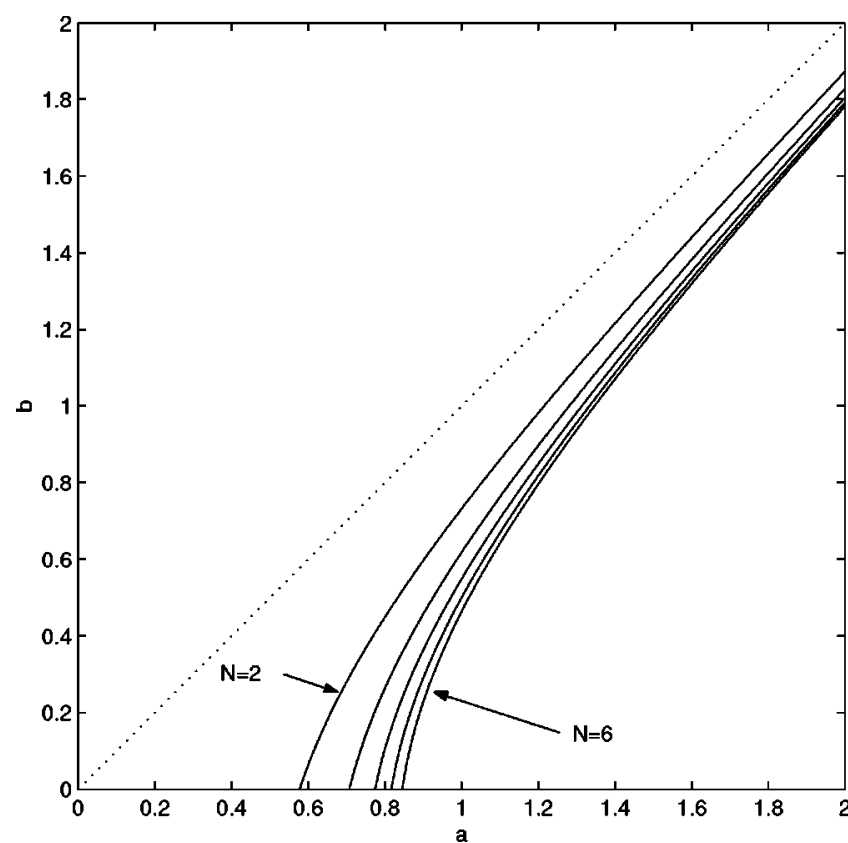

FIG. 1. $b$ as a function of $a$ for $N=2,3,4,5$ and 6 . The curves begin at $a=a_{\text {crit }}(N)$ as given in (19) and asymptote to the dotted line as $a \rightarrow \infty$.

A graph of the solution for $b$ is given in Fig. 1 as a function of $a$ for $a \geqslant a_{\text {crit }}(N)$ for $N=2,3,4,5$, and 6. A similar graph of $\Omega_{\max }(a, N)$ against $a$ is shown in Fig. 2 for the same values of $N$. To characterize the solutions, a more natural (and more physical) choice of parameter than $a$ (which has been introduced for purely mathematical reasons) is to parametrize them by the value of $N$ and $\Omega_{\max }(a, N)$. In Fig. 2 it is clear from the monotonicity of $\Omega_{\max }(a, N)$ as a function of $a$ that such parameters specify a unique solution.

\section{B. The limits $a=a_{\text {crit }}(N)$ and $a \rightarrow \infty$ for fixed $N$}

It is of interest to examine the two limits $a=a_{\text {crit }}(N)$ and $a \rightarrow \infty$ for fixed values of $N$ :
Limit $a=a_{\text {crit }}(N)$ : When $a=a_{\text {crit }}(N), b=0$. This means that $\psi$ is purely a function of $|z|^{2}$ so that the streamlines are just circles centered at $z=0$. This is the analog of the $C=1$ limit of Stuart's solution (3), which yields a homogeneous shear layer profile with unidirectional streamlines.

Line vortex limit: $a \rightarrow \infty$ : Now fix a value of $N$ and consider the limit $a \rightarrow \infty$. Note from (18) that $b \rightarrow a$ as $a \rightarrow \infty$ so that

$$
\psi \sim-\frac{1}{2} \log \left[\frac{N^{2} z^{N-1} \bar{z}^{N-1}}{\left(z^{N}+1\right)^{2}\left(\bar{z}^{N}+1\right)^{2}}\right], \quad \text { as } a \rightarrow \infty,
$$

which is irrotational almost everywhere except for line vortex singularities at $z=0$ and at the $N$ roots of $z^{N}+1=0$. Let the circulation of the line vortex at $z=0$ be $\Gamma_{0}$ and that of the $N$ satellite line vortices be $\Gamma_{s}$ (it is clear from symmetry that they all have the same circulation). Then using the fact that a line vortex at $z_{0}$ of circulation $\Gamma$ is

$$
\psi=-\frac{\Gamma}{4 \pi} \log \left(\left(z-z_{0}\right)\left(\bar{z}-\bar{z}_{0}\right)\right),
$$

we recognize that

$$
\Gamma_{0}=2 \pi(N-1), \quad \Gamma_{s}=-4 \pi \text {. }
$$

As a check on the above, Morikawa and Swenson ${ }^{9}$ have considered a polygonal array of $N$ satellite line vortices of circulation $\gamma_{s}$ with a central line vortex of circulation $\gamma_{0}$. For general values of the ratio $\gamma_{0} / \gamma_{s}$, such a configuration is a relative equilibrium of the planar Euler equations in which the polygonal array of satellite vortices rotates about the central line vortex with constant angular velocity. For a special choice of the ratio of central-to-satellite vortex circulations the angular velocity vanishes and the configuration is stationary. A simple exercise in writing down the relevant complex potential and applying the condition that the local non-selfinduced component of the velocity field at any of the satellite

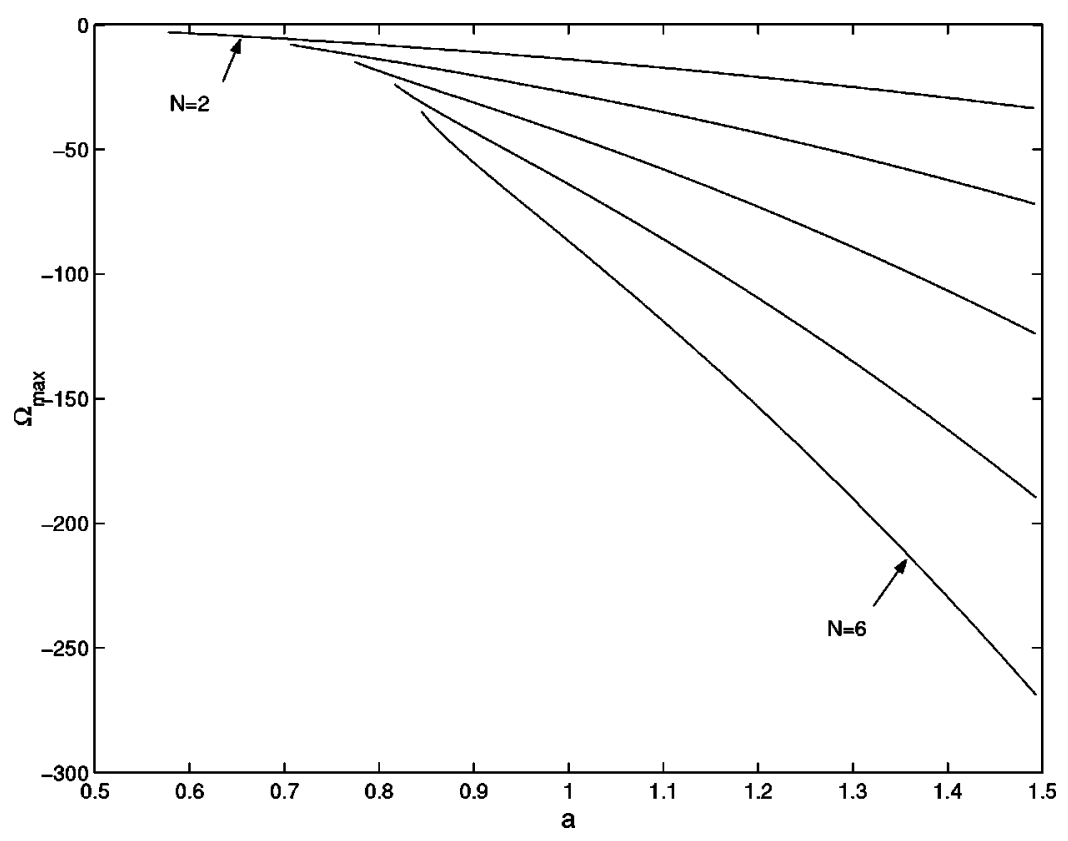

FIG. 2. $\Omega_{\max }$ as a function of $a$ for $N=2,3,4,5$ and 6 . The curves begin at $a=a_{\text {crit }}(N)$ as given in (19). 


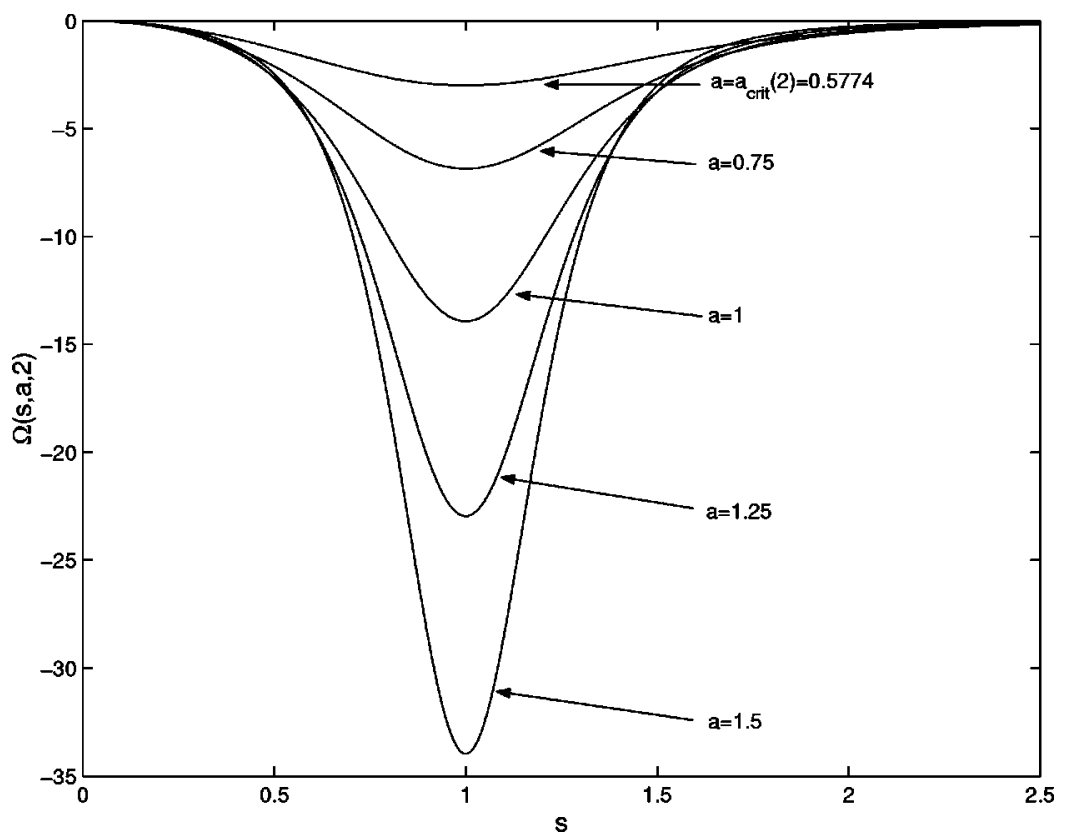

FIG. 3. The vorticity profile $\Omega(s, a, 2)$ along the imaginary axis as a function of $s$ for various choices of $a$. The profile becomes more peaked about the minimum value $\Omega_{\max }(a, 2)$ with increasing $a$ (tending to a $\delta$-function distribution as $a \rightarrow \infty)$.

line vortex positions is zero shows that this special ratio is $-(N-1) / 2$. This is consistent with the results (26). Therefore, the $a \rightarrow \infty$ limit of the smooth Stuart vortex solution found here is precisely the stationary case of the point vortex problem considered by Morikawa and Swenson. ${ }^{9}$

\section{General case: $a_{\text {crit }}(N)<a<\infty$}

For general values of $a_{\text {crit }}(N)<a<\infty$, the solutions describe a stationary line vortex of circulation $2 \pi(N-1)$ at the origin surrounded by an everywhere-smooth distribution of negative vorticity characterized by $N$ vorticity extrema symmetrically-disposed about the origin. Figure 3 shows a graph of $\Omega(s, a, 2)$ - the vorticity profile along the ray $\arg [z]=\pi / 2$ - for various choices of $a$. It is clear that as $a$ increases, the vorticity profile becomes more peaked and more concentrated about the extremum. Figures 4-7 show typical distributions of streamlines (i.e., the $\psi$-contours) associated with the $a=1$ solutions with $N=2$ (a tripole), $N$ $=3$ (a quadrupole), $N=4$ (a pentapole) and $N=10$ (a "decapole"). The Kelvin cat's-eye patterns, familiar from the Stuart solution ${ }^{17}$ for an infinite row of vortices, are clear.

It is natural to consider the total circulation associated with each satellite vortex relative to the circulation $\Gamma_{0}$ $=2 \pi(N-1)$ associated with the central point vortex. Given the $N$-polygonal symmetries of the vortex configuration, it is natural to define the circulation $\widetilde{\Gamma}_{s}$ to be the total vorticity in a sector $S_{N}$ defined as

$$
S_{N} \equiv\left\{z \in \mathbb{C}\left|0 \leqslant \arg [z]<\frac{2 \pi}{N},\right| z \mid \neq 0\right\},
$$

so that

$$
\tilde{\Gamma}_{s} \equiv \iint_{S_{N}} \omega d x d y
$$

Here it is understood that $\omega$ denotes the smooth vorticity distribution for points $z \neq 0$ so that we do not take into account the $\delta$-function distribution of vorticity at the origin. Interestingly, it can be shown that

$$
\widetilde{\Gamma}_{s}=-4 \pi
$$

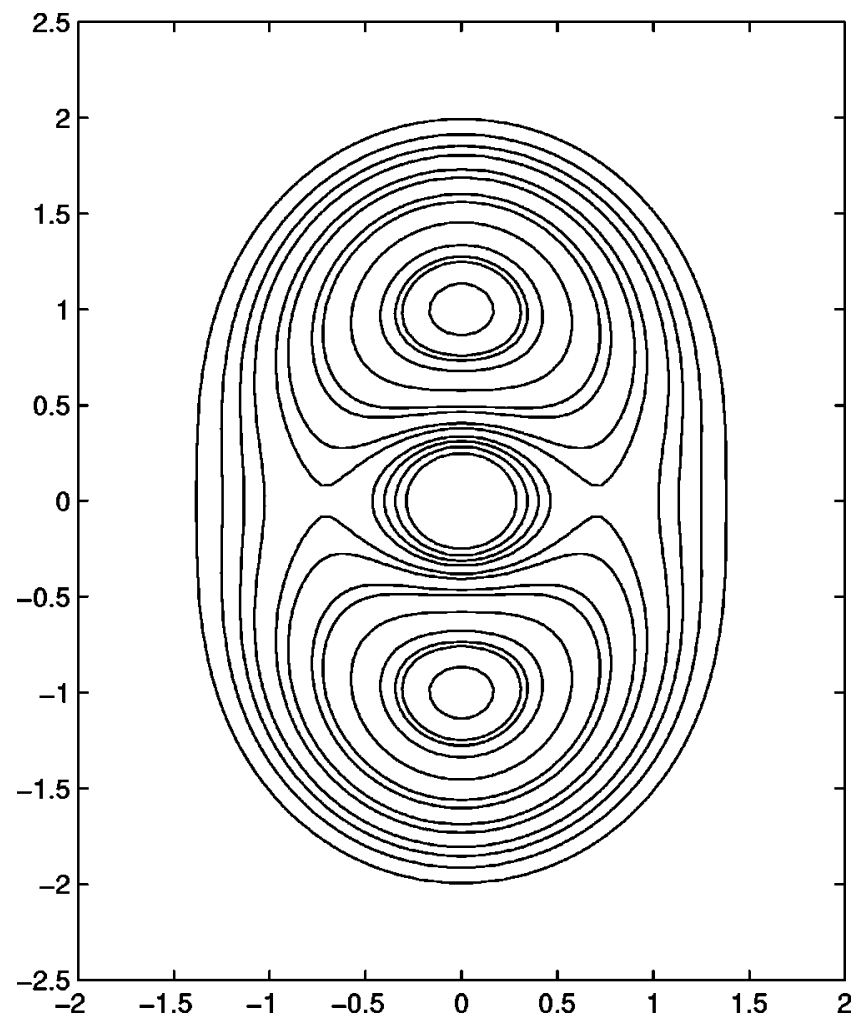

FIG. 4. Streamlines and iso-vorticity contours of a tripole: $N=2, a=1$, $\Omega_{\max }=-13.928$ (to 3 decimal places). The vorticity is everywhere smooth except for a point vortex of circulation $2 \pi$ at the origin. 


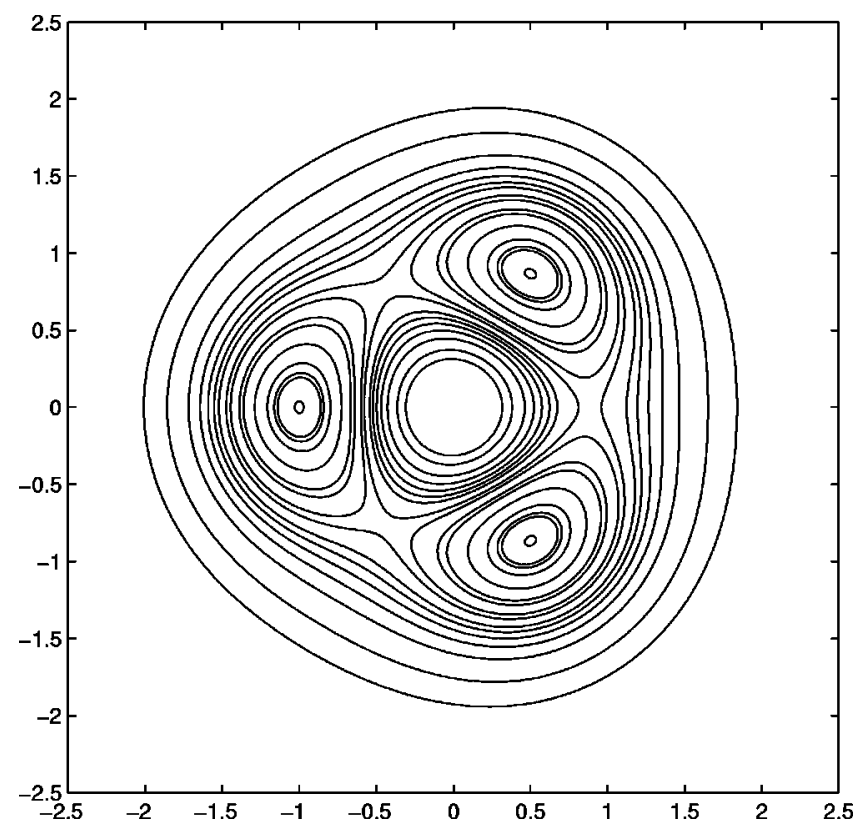

FIG. 5. Streamlines and iso-vorticity contours of a quadrupole: $N=3, a$ $=1, \Omega_{\max }=-27.416$. The vorticity is everywhere smooth except for a point vortex of circulation $4 \pi$ at the origin.

independent of the choice of either $a$ or $N$. Thus, changing $a$ simply adjusts the distribution of vorticity within the sector $S_{N}$ while maintaining the same total circulation $\widetilde{\Gamma}_{s}$ there. This result is established in Appendix B. It is consistent with the point-vortex limit considered in the previous section. Stuart ${ }^{17}$ observed an analogous phenomenon in respect of his solution (3): the total circulation inside any period of the periodic array of vortices is always $-4 \pi$ independent of the value of the parameter $C$; only the distribution of vorticity within each period changes with $C$.

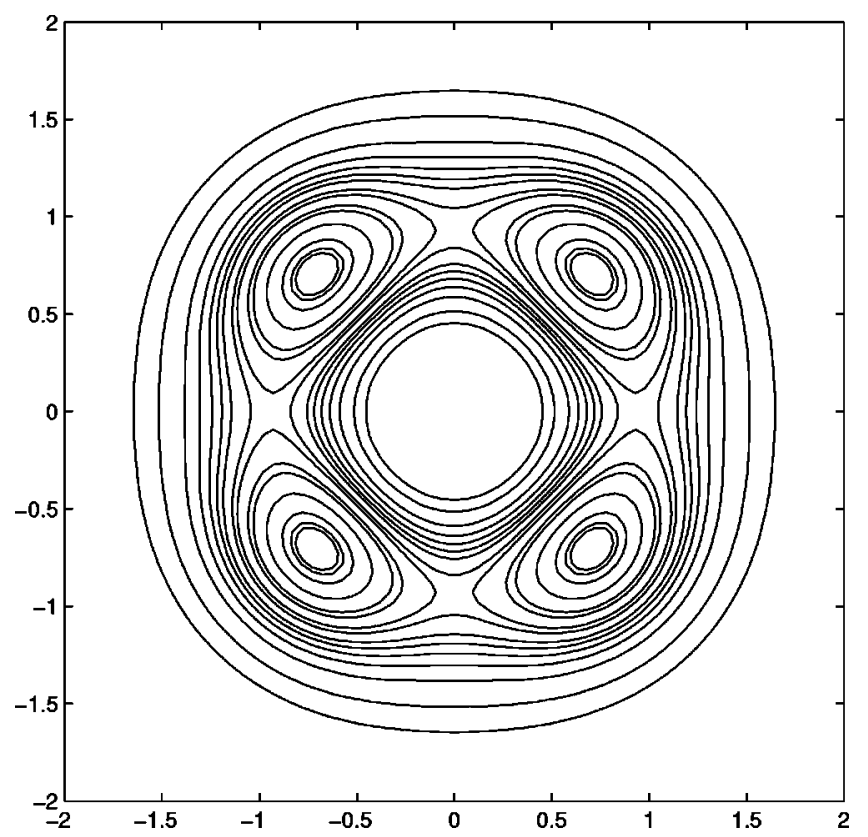

FIG. 6. Streamlines and iso-vorticity contours of a pentapole: $N=4, a=1$, $\Omega_{\max }=-44.166$. The vorticity is everywhere smooth except for a point vortex of circulation $6 \pi$ at the origin.

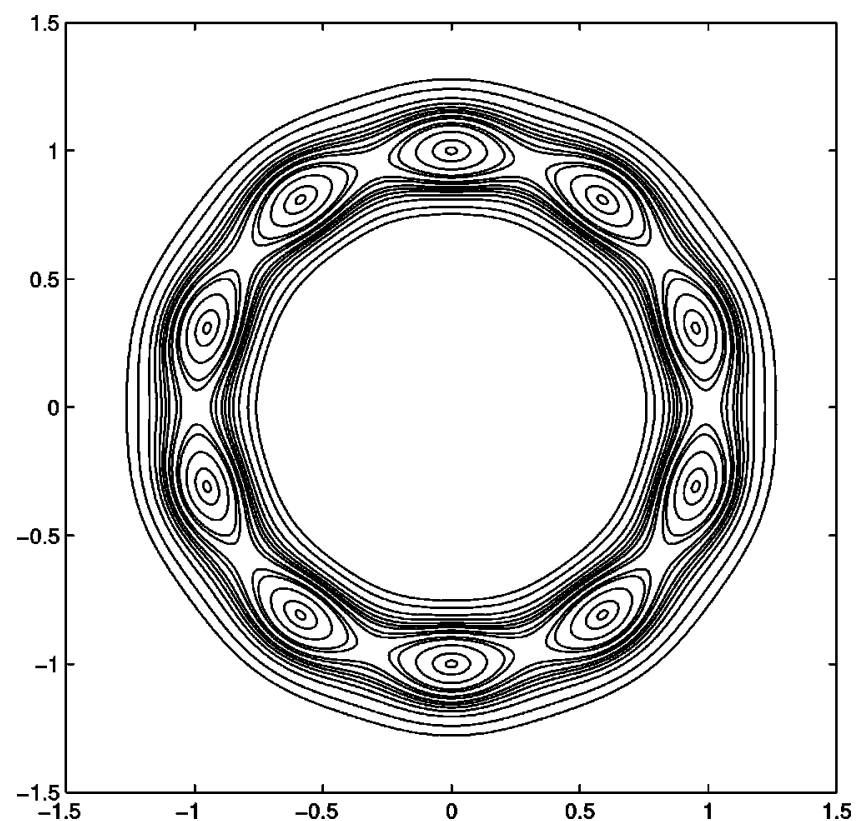

FIG. 7. Streamlines and iso-vorticity contours of a decapole: $N=10, a$ $=1, \Omega_{\max }=-206.178$. The vorticity is everywhere smooth except for a point vortex of circulation $18 \pi$ at the origin.

\section{The limit $N \rightarrow \infty$ for fixed $a$}

There is another interesting limit to consider in respect of this new class of exact solutions, namely $N \rightarrow \infty$. It should be clear that as $N$ is increased, so that the number of vorticity extrema equispaced about the unit circle gets larger, on a length-scale that is small with respect to unity (the radius of curvature of this circular ring of cat's-eye vortices) the solution will locally resemble a periodic street of smooth vortices in the spirit of Stuart's original solution ${ }^{17}$ for an infinite periodic array of vortices. This asymptotic limit is now examined in detail.

Note first, from (18), that

$$
b \rightarrow a, \quad \text { as } N \rightarrow \infty .
$$

On a length-scale of $\mathcal{O}(1 / N)$ near the point $z=1$, as $N \rightarrow \infty$ we expect the solution to locally resemble the Stuart vortex solution for an infinite row of vortices extending in a direction parallel to the imaginary axis. For this reason, we introduce a rescaled complex variable $\zeta$ defined by

$$
z=1+\frac{i}{N} \zeta
$$

where the $i$ accounts for the fact that the street of vortices locally appears parallel to the imaginary axis and the $1 / N$ factor means that the solution (9) will resemble the Stuart vortex layer for $\zeta=\mathcal{O}(1)$. To verify this, note that

$$
\lim _{N \rightarrow \infty}\left[z^{N}\right]=\lim _{N \rightarrow \infty}\left(1+\frac{i \zeta}{N}\right)^{N}=e^{i \zeta}
$$

Similarly,

$$
\lim _{N \rightarrow \infty}\left[z^{N-1}\right]=e^{i \zeta} .
$$


Substituting (32) and (33) into the solution (9) in the limit $N \rightarrow \infty$, to within constants, the limit of the solution is

$$
\begin{aligned}
\psi \sim & -\frac{1}{2} \log \left[\frac{e^{i \zeta} e^{-i \bar{\zeta}}}{\left(1+a^{2}\left(e^{i \zeta}+1\right)\left(e^{-i \bar{\zeta}}+1\right)\right)^{2}}\right]+\text { const } \\
= & \log \left[e^{-i \zeta / 2} e^{i \bar{\zeta} / 2}+a^{2}\left(e^{i \zeta / 2}+e^{-i \zeta / 2}\right)\left(e^{i \bar{\zeta} / 2}+e^{-i \bar{\zeta} / 2}\right)\right]+\text { const } \\
= & \log \left[\left(1+4 a^{2}\right) \cos (\zeta / 2) \cos (\bar{\zeta} / 2)+\sin (\zeta / 2) \sin (\bar{\zeta} / 2)\right. \\
& -i \sin (\zeta / 2) \cos (\bar{\zeta} / 2)+i \sin (\bar{\zeta} / 2) \cos (\zeta / 2)]+ \text { const. }
\end{aligned}
$$

By modifying the analysis of Appendix A, it can be shown that, to within an appropriate choice of the inconsequential constant $c$, the solution (34) is equivalent to taking

$$
f(\zeta)=A \tan (\zeta / 2)+B,
$$

with $A=1 / 2 a$ and $B=i / 2 a$, in (6). This is essentially the Stuart vortex solution, as discussed in Appendix A.

\section{DISCUSSION}

A class of exact solutions having smooth distributed vorticity and corresponding to stationary polygonal arrays of vortices with $N$-fold symmetry has been constructed. The solutions can be understood to be "smoothed-out" versions of the stationary line vortex configurations considered by Morikawa and Swenson. ${ }^{9}$ At the same time, they can alternatively be understood as finite $N$-vortex counterparts to the $\infty$-vortex Stuart layer solution. ${ }^{17}$

The specific choice of rational function $f(z)$ given in (8) has been shown to correspond to smooth $N$-polygonal arrays of vortices centered around a point vortex at the origin. The configurations have been shown to be equilibria of the Euler equations. It should be clear that other choices of rational function $f(z)$ are possible, in principle. However, care must be taken in postulating other choices. In general, arbitrary choices of rational function $f(z)$, even ones possessing just simple pole singularities in $\mathrm{C}$ (which do not result in singular points of the solution), will necessarily have a distribution of zeros of $f^{\prime}(z)$ in C. Such zeros will yield singularities of $\psi$ corresponding to point vortex singularities. While these are physically admissible, each must be stationary under the effects of the local non-self-induced velocity field if the solution is to be a consistent global equilibrium of the Euler equations. Such conditions will impose constraints on the poles and zeros of the rational function $f(z)$. If and only if a choice of $f(z)$ can be made at which all such conditions are satisfied, then a consistent steady solution will result.

Pierrehumbert and Widnall ${ }^{21}$ have studied the linear stability of the planar Stuart vortex solution. The stability of the new $N$-polar solutions found here is of interest but is beyond the scope of the present paper, requiring a detailed analysis. Recall, however, that the $a \rightarrow \infty$ limit of the solutions corresponds to the stationary point vortex configurations considered by Morikawa and Swenson, ${ }^{9}$ and Table 1 of Ref. 9 gives the range of values of $\Gamma_{0} / \Gamma_{s}$ for which the line-vortex equilibria are linearly stable for different values of $N$. As previously discussed, in the limit $a \rightarrow \infty$ the solutions here correspond to the ratio $\Gamma_{0} / \Gamma_{s}=-(N-1) / 2$. Inspection reveals that only in the case $N=3$, i.e., in the case of "quadrupolar vortices," does this ratio lie in the linear stability range computed by Morikawa and Swenson. ${ }^{9}$ It will be of interest to examine whether these linear stability properties are shared by the smooth $a<\infty$ solutions. It is also worth mentioning that short-wave instability studies of the planar Stuart vortices in a rotating frame have been carried out by Godeford, Cambon, and Leblanc ${ }^{22}$ (see also Sipp and Jacquin ${ }^{23}$ ) and such techniques might well be of use in analyzing the stability properties of the present class of solutions.

Given the exact solutions, additional physical effects can be added either numerically or perturbatively. For example, it is reasonable to suggest that there is a smooth connected set of solutions consisting of vortex arrays with similar vorticity distributions that are rotating. The structure of such rotating vortex arrays might be studied using a perturbation analysis about the exact solutions found herein.

\section{ACKNOWLEDGMENTS}

This work was carried out while the author was a Visiting Associate Professor in the Mathematics Department at MIT during the spring semester 2003. It is partially supported by a grant from the Engineering and Physical Sciences Research Council of the U.K.

\section{APPENDIX A: STUART'S (1967) SOLUTION}

Stuart's solution (3) solves (4) with $\widetilde{c}=1, d=-2$ (note that this corresponds to $c=\widetilde{c} / 4=1 / 4)$. Stuart ${ }^{17}$ records that (3) can be derived from (6) by the particular choice

$$
f(z)=A \tan (z / 2) \text {. }
$$

Then

$$
\psi=\log \left(\frac{1}{A} \cos (z / 2) \cos (\bar{z} / 2)+A \sin (z / 2) \sin (\bar{z} / 2)\right) .
$$

With the use of

$$
\begin{aligned}
& \cos (z / 2) \cos (\bar{z} / 2)=\frac{1}{2}(\cosh y+\cos x), \\
& \sin (z / 2) \sin (\bar{z} / 2)=\frac{1}{2}(\cosh y-\cos x),
\end{aligned}
$$

(A2) becomes

$$
\psi=\log \left(\cosh y\left(\frac{1}{2 A}+\frac{A}{2}\right)+\cos x\left(\frac{1}{2 A}-\frac{A}{2}\right)\right) .
$$

Exploiting the fact that if

$$
C \equiv \frac{1}{2 A}+\frac{A}{2},
$$

and $0 \leqslant A \leqslant 1$ then

$$
\frac{1}{2 A}-\frac{A}{2}=\sqrt{C^{2}-1},
$$

(A4) is precisely Stuart's solution (3). It is noted that if the choice (A1) is modified by the addition of a constant, i.e.,

$$
f(z)=A \tan (z / 2)+B,
$$


the resulting solution is qualitatively the same as the solution given by Stuart's particular choice (A1).

\section{APPENDIX B: CIRCULATION OF SATELLITE VORTICES}

In this appendix, it is shown that the circulation $\widetilde{\Gamma}_{s}$ associated with each satellite vortex [as defined in (28)] is $-4 \pi$ irrespective of the choice of either $N$ or $a$. With the use of (28), (1) and (9), we obtain

$$
\omega=-\frac{4 N^{2} a^{2} z^{N-1} \bar{z}^{N-1}}{\left(1+\left(a z^{N}+b\right)\left(a \bar{z}^{N}+b\right)\right)^{2}} .
$$

Using the standard wedge product (see, for example, Sec. 7.6 of Ref. 24) defined so that $d z \wedge d \bar{z}=-2 i d x d y$, we can write (28) as

$$
\widetilde{\Gamma}_{s}=-\frac{1}{2 i} \iint_{S_{N}}-\frac{4 a^{2}\left(N z^{N-1}\right)\left(N \bar{z}^{N-1}\right) d z \wedge d \bar{z}}{\left(1+\left(a z^{N}+b\right)\left(a \bar{z}^{N}+b\right)\right)^{2}} .
$$

It is now convenient to change the variable to integration to

$$
w \equiv z^{N} .
$$

The domain of integration then becomes the entire complex $w$-plane, denoted C [ this is the image of the domain $S_{N}$ under the conformal map (B3)]. Using the fact that $d w$ $=N z^{N-1} d z$, we obtain

$$
\widetilde{\Gamma}_{s}=\frac{1}{2 i} \iint \frac{4 a^{2} d w \wedge d \bar{w}}{(1+(a w+b)(a \bar{w}+b))^{2}} .
$$

Now observe that

$$
\begin{gathered}
\frac{\partial}{\partial \bar{w}}\left(\frac{a \bar{w}+b}{(1+(a w+b)(a \bar{w}+b))}\right) \\
=\frac{a}{(1+(a w+b)(a \bar{w}+b))^{2}},
\end{gathered}
$$

so that (B4) can be written as

$$
\Gamma_{s}=\frac{1}{2 i} \iint_{\mathrm{C}} 4 a \frac{\partial}{\partial \bar{w}}\left(\frac{a \bar{w}+b}{(1+(a w+b)(a \bar{w}+b))}\right) d w \wedge d \bar{w} .
$$

The complex form of Green's theorem (Sec. 7.6 of Ref. 24) takes the form

$$
\iint_{D} \frac{\partial \Phi(w, \bar{w})}{\partial \bar{w}} d w \wedge d \bar{w}=-\oint_{\partial D} \Phi(w, \bar{w}) d w
$$

where $\Phi(w, \bar{w})$ is some nonsingular function in a domain $D$ and $\partial D$ is the boundary of this domain. Using this result in (B6) yields that

$$
\begin{aligned}
\tilde{\Gamma}_{s} & =-\frac{2 a}{i} \lim _{R \rightarrow \infty}\left[\oint_{|w|=R}\left(\frac{a \bar{w}+b}{1+(a w+b)(a \bar{w}+b)}\right) d w\right] \\
& =-\frac{2 a}{i} \lim _{R \rightarrow \infty}\left[\int_{\theta=0}^{2 \pi} \frac{\left(a R e^{-i \theta}+b\right) i R e^{i \theta} d \theta}{1+\left(a R e^{i \theta}+b\right)\left(a R e^{-i \theta}+b\right)}\right] \\
& =-4 \pi .
\end{aligned}
$$

${ }^{1}$ P. K. Newton, The N-Vortex Problem (Springer-Verlag, New York, 2001).

${ }^{2}$ P. G. Saffman, Vortex Dynamics (Cambridge University Press, Cambridge, 1992).

${ }^{3}$ J. J. Thomson, A Treatise on the Motion of Vortex Rings (Macmillan, London, 1883)

${ }^{4} \mathrm{~T}$. H. Havelock, "The stability of motion of rectilinear vortices in ring formation," Philos. Mag. 1, 617 (1931).

${ }^{5}$ E. J. Yarmchuk, M. J. V. Gordon, and R. E. Packard, "Observation of stationary vortex arrays in rotating superfluid helium," Phys. Rev. Lett. 43, 214 (1979).

${ }^{6}$ L. J. Campbell and R. M. Ziff, “A catalog of 2-D vortex patterns," Los Alamos Sci. Report, LA-7384-MS, 1978.

${ }^{7}$ D. A. Schecter, D. H. E. Dubin, K. S. Fine, and A. C. Cass, "Vortex crystals from 2D Euler flow: Experiment and simulation," Phys. Fluids 11, 905 (1999).

${ }^{8}$ D. Durkin and J. Fajans, "Experiments of two-dimensional vortex patterns," Phys. Fluids 12, 289 (2000)

${ }^{9}$ G. K. Morikawa and E. V. Swenson, "Interacting motion of rectilinear geostrophic vortices," Phys. Fluids 14, 1058 (1971).

${ }^{10}$ Y. G. Morel and X. J. Carton, "Multipolar vortices in two-dimensional incompressible flows," J. Fluid Mech. 267, 23 (1994).

${ }^{11}$ G. F. Carnevale and R. Kloosterziel, "Emergence and evolution of triangular vortices," J. Fluid Mech. 259, 305 (1994).

${ }^{12}$ D. G. Crowdy, "A class of exact multipolar vortices," Phys. Fluids 11, 2556 (1999).

${ }^{13} \mathrm{H}$. Aref and D. L. Vainchtein, "Point vortices exhibit asymmetric equilibria," Nature (London) 392, 769 (1998).

${ }^{14}$ D. G. Dritschel, "The stability and energetics of co-rotating uniform vortices," J. Fluid Mech. 157, 95 (1985).

${ }^{15}$ D. G. Crowdy, "Exact solutions for rotating vortex arrays with finite-area cores," J. Fluid Mech. 469, 209 (2002).

${ }^{16}$ V. V. Meleshko and G. J. F. van Heijst, “On Chaplygin's investigations of two-dimensional vortex structures in an inviscid fluid," J. Fluid Mech. 272, 157 (1994)

${ }^{17}$ J. T. Stuart, "On finite amplitude oscillations in laminar mixing layers," J. Fluid Mech. 29, 417 (1967).

${ }^{18}$ R. Mallier and S. A. Maslowe, "A row of counter-rotating vortices," Phys. Fluids A 5, 1074 (1993).

${ }^{19}$ D. G. Crowdy, "General solutions to the 2D Liouville equation," Int. J. Eng. Sci. 35, 141 (1997).

${ }^{20}$ H. Lamb, Hydrodynamics, 6th ed., Cambridge University Press, Cambridge (1932).

${ }^{21}$ R. T. Pierrehumbert and S. E. Widnall, "The two and three-dimensional instabilities of a spatially periodic shear layer," J. Fluid Mech. 144, 59 (1982)

${ }^{22}$ F. S. Godeford, C. Cambon, and S. Leblanc, "Zonal approach to centrifugal, elliptic, and hyperbolic instabilities in Stuart vortices with external rotation," J. Fluid Mech. 449, 1 (2001).

${ }^{23}$ D. Sipp and L. Jacquin, "Three-dimensional centrifugal-type instabilities of two-dimensional flows in rotating systems," Phys. Fluids 12, 1740 (2000).

${ }^{24}$ A. S. Fokas and M. J. Ablowitz, Complex Variables (Cambridge University Press, Cambridge, 1997). 